\title{
Histoire de l'art et anthropologie des techniques. Objets, processus, représentations
}

Art history and the anthropology of techniques. Objects, processes, representations

Philippe Cordez

\section{(2) OpenEdition \\ Journals}

Electronic version

URL: https://journals.openedition.org/tc/8922

DOI: $10.4000 /$ tc. 8922

ISSN: 1952-420X

Publisher

Éditions de l'EHESS

Electronic reference

Philippe Cordez, "Histoire de l'art et anthropologie des techniques. Objets, processus, représentations", Techniques \& Culture [Online], Varia, Online since 15 January 2019, connection on 29 September 2022. URL: http://journals.openedition.org/tc/8922 ; DOI: https://doi.org/10.4000/tc.8922

This text was automatically generated on 29 September 2022.

All rights reserved 


\title{
Histoire de l'art et anthropologie des techniques. Objets, processus, représentations
}

\author{
Art history and the anthropology of techniques. Objects, processes, \\ representations
}

Philippe Cordez

Lorsque les mots « outil » et « instrument » apparurent en français au XII siècle, leur signification était similaire: ils désignaient les objets que les humains fabriquent en vue d'exécuter une tâche ${ }^{1}$. Or ces termes ont si bien divergé qu'ils correspondent aujourd'hui à deux genres distincts - ou plus exactement, à deux champs d'activités. Les outils sont, dans le langage actuel, des choses qui peuvent être tenues en main pour exercer une action sur un matériau brut, comme le bois, la pierre ou le métal, dans le but de le mettre en forme. Ils sont également conçus pour travailler sur une pièce déjà aboutie, comme une moto. Les instruments ont une fonction similaire, mais sont plus finement élaborés et réservés à des domaines privilégiés, comme la musique, la chirurgie ou les sciences. Cette différenciation s'est particulièrement imposée au XIX ${ }^{\mathrm{e}}$ siècle: les artistes, en particulier les peintres, travaillent alors avec des instruments, les outils étant communément attribués aux ouvriers ${ }^{2}$. À cela vient s'ajouter la longue histoire des notions de machine ou d'appareil, qui renvoient à d'autres objets et domaines d'activité, relevant cette fois de la mécanique et de l'électronique.

1 Ces observations, ouvrent un vaste chantier pour l'histoire de l'art. Depuis sa constitution en discipline académique, celle-ci est elle-même marquée par les enjeux de la différenciation entre outils et instruments. À partir de ces deux mots qui définissent des champs d'activités distincts, il semble possible d'éclairer certains aspects de l'histoire de la fabrication et du maniement des objets dans les sociétés occidentales, et en particulier de mieux saisir la place respective de ce qui y est désigné en tant qu' « art » et en tant que « technique ». Plus largement, se pose la question de l'apport de l'histoire de l'art aux recherches sur les techniques. 
2 Une telle démarche implique de prendre en considération un important spectre d'outils et d'instruments historiques qui, bien qu'ils ne soient pas des œuvres d'art au sens usuel du terme, n'en sont pas moins des objets élaborés liés à des savoir-faire spécialisés. Les travaux sur les fonctions techniques des images ont récemment ouvert une piste ${ }^{3}$ qui peut être élargie aux objets, en explorant comment leurs matières et leurs formes participent de processus artistiques et techniques. Outils et instruments étant enfin impliqués dans les représentations visuelles de tels actes, ils jouent de fait un rôle majeur dans la socialisation des arts et des techniques.

\section{Objets}

Outils et instruments ne sont pas, au sens strict, des objets: comme le suggère l'étymologie d'objectum, mot qui dérive d'objicere (jeter devant), les objets se définissent par rapport à des sujets dont ils affectent les sens. Ils sont distincts de ceux-ci, mis à distance et non actifs. Au contraire, on se représente avant tout les outils et les instruments comme impliqués dans un acte et en relation physique directe avec un sujet. Ceci est évident par exemple dans l'image mentale que l'on aurait d'un lasso : ce qui vient d'abord à l'esprit n'est pas la corde figée, enroulée autour d'un clou fixé au mur, mais sa forme en mouvement, animée dans l'air par le geste du cow-boy. Dès lors qu'on les expose et qu'on les classe, outils et instruments sont pourtant immobilisés, privés de leurs fonctions premières, et finalement constitués en tant qu'objets de curiosité ou de science. Ceci invite à parcourir l'histoire des collections où ils furent conservés, en portant à chaque fois une attention particulière au statut qui leur a été conféré.

3 Les cabinets de curiosités de l'époque moderne constituent à cet égard un premier type de situation. Dans un traité publié en 1565, Samuel Quiccheberg proposait d'y rassembler notamment des instruments (instrumenta) de musique et de mathématiques avec d'autres destinés à l'écriture et à la peinture, aux arts "plus artistiques » ( $a b$ artificiosoribus artificibus usurpantur) ou communs (omnium artificum), jusqu'à la chirurgie, à la chasse ou aux jeux ${ }^{4}$. Nul doute que semblable réunion en des lieux spécifiques eut des conséquences sur la manière dont on les maniait et dont on les comprenait. Le banc à tréfiler fabriqué pour l'usage personnel du Prince-électeur Auguste de Saxe (1553-1586) n'a ainsi que peu à voir avec l'instrumentum d'un artisan plus ordinaire. De par son ornementation complexe et précieuse de marqueterie et de métal gravé à l'eau forte, et en raison de sa mécanique ostensiblement puissante (avec une crémaillère et un boîtier de manivelle innovants), cet objet participait de la mise en scène des capacités du souverain ${ }^{5}$. Parallèlement, les premières collections d'instruments musicaux apparues durant la deuxième moitié $\mathrm{du} \mathrm{xVI}^{\mathrm{e}}$ siècle sont directement liées au développement d'une nouvelle musique instrumentale. Celle-ci s'établit alors comme un genre propre à côté de la musique vocale tout en permettant, dans le cas de Dresde, à Michael Praetorius, qui fut le maître de chapelle du Princeélecteur de Saxe, de publier vers 1620 la première description systématique et analytique d'instruments de musique ${ }^{6}$. Dans les milieux de cour du XvII ${ }^{\mathrm{e}}$ siècle, on développa une nouvelle instrumentation scientifique venant s'ajouter aux compas, règles, balances, astrolabes ou bien encore aux horloges qui existaient déjà. Ainsi les télescopes et les microscopes, les thermomètres et les baromètres, destinés à des expériences par lesquelles on aspirait à mieux comprendre le monde, et qui suscitaient 
en même temps l'intérêt pour eux-mêmes ${ }^{7}$. Cette fascination de l'époque moderne pour les objets fonctionnels ${ }^{8}$ entraîna d'autres fabrications, dont le but évident était de mener la notion d'instrument jusqu'au point de la non-fonctionnalité tout en se jouant de cette limite. En témoigne par exemple une sphère en pietra paesina de Toscane que le savant danois Ole Worm, tirant parti de la structure de cette pierre verte et brune qui évoque les lignes complexes des conventions cartographiques, fit monter vers 1650 à la manière d'un globe terrestre 9 . Ou bien un clavecin, sculpté en 1687 dans du marbre de Carrare pour le duc François II d'Este à Modène, et sur lequel on pouvait jouer pour de vrai ${ }^{10}$.

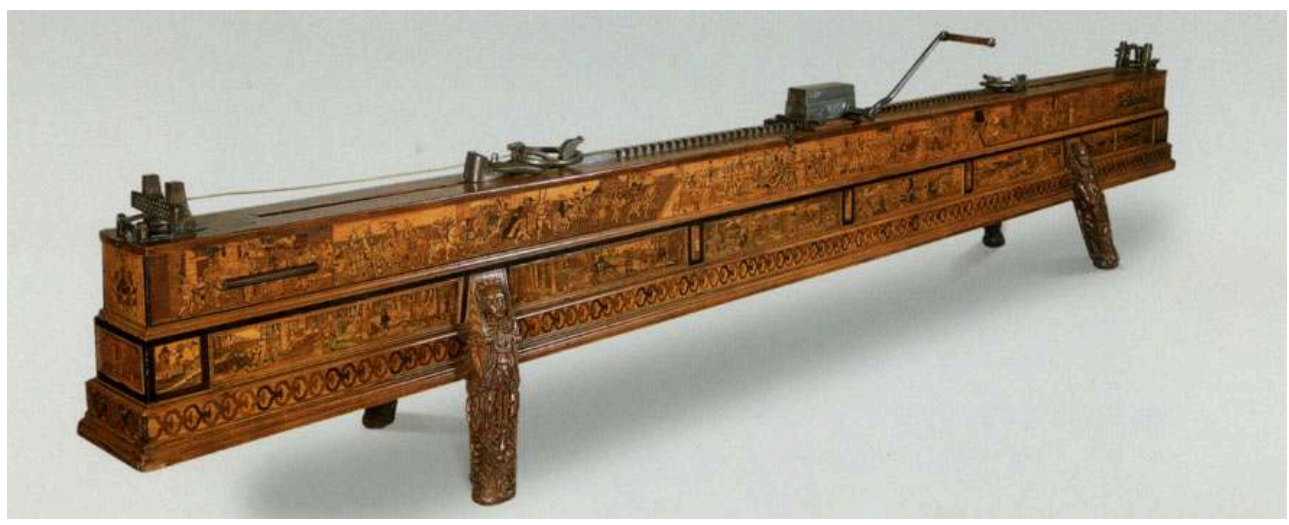

Banc à tréfiler du prince-électeur Auguste de Saxe, Leonhard Danner, 1565. Écouen, Musée national de la Renaissance.

Cliché : Welker 2002 : 119.

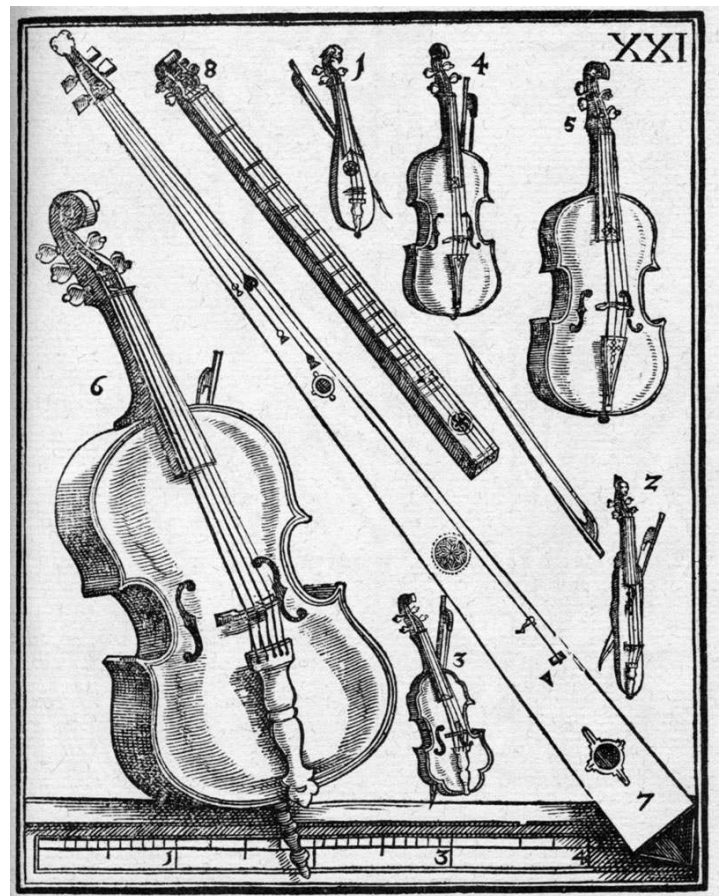

2. 2. Fictine Pofchen / Geigen em Octar böber. 3. Difcant-Beig en Quart böber.

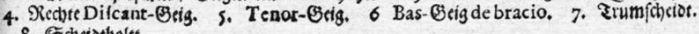
Schciotholtt.

E $\quad$ ifj

Praetorius, M. Syntagma Musicum, 2, De Organographia, Wolfenbüttel : Holwein 1619, pl. xxı. Fac-similé, Kassel etc. : Bärenreiter, 1958.

Bibliothèque nationale de France. 


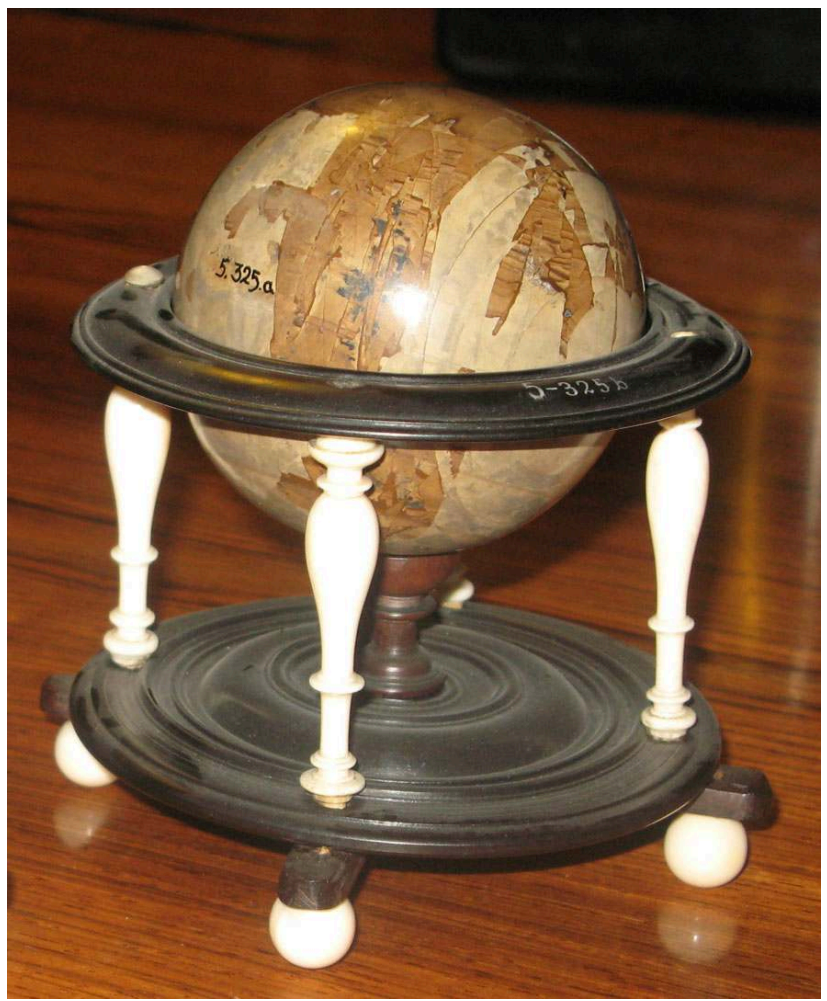

Boule de pietra paesina, montée comme un globe terrestre pour Ole Worm, vers 1650. Copenhague, château de Rosenborg, Kongens Kunstkammer.

Cliché : Lisbet Tarp.

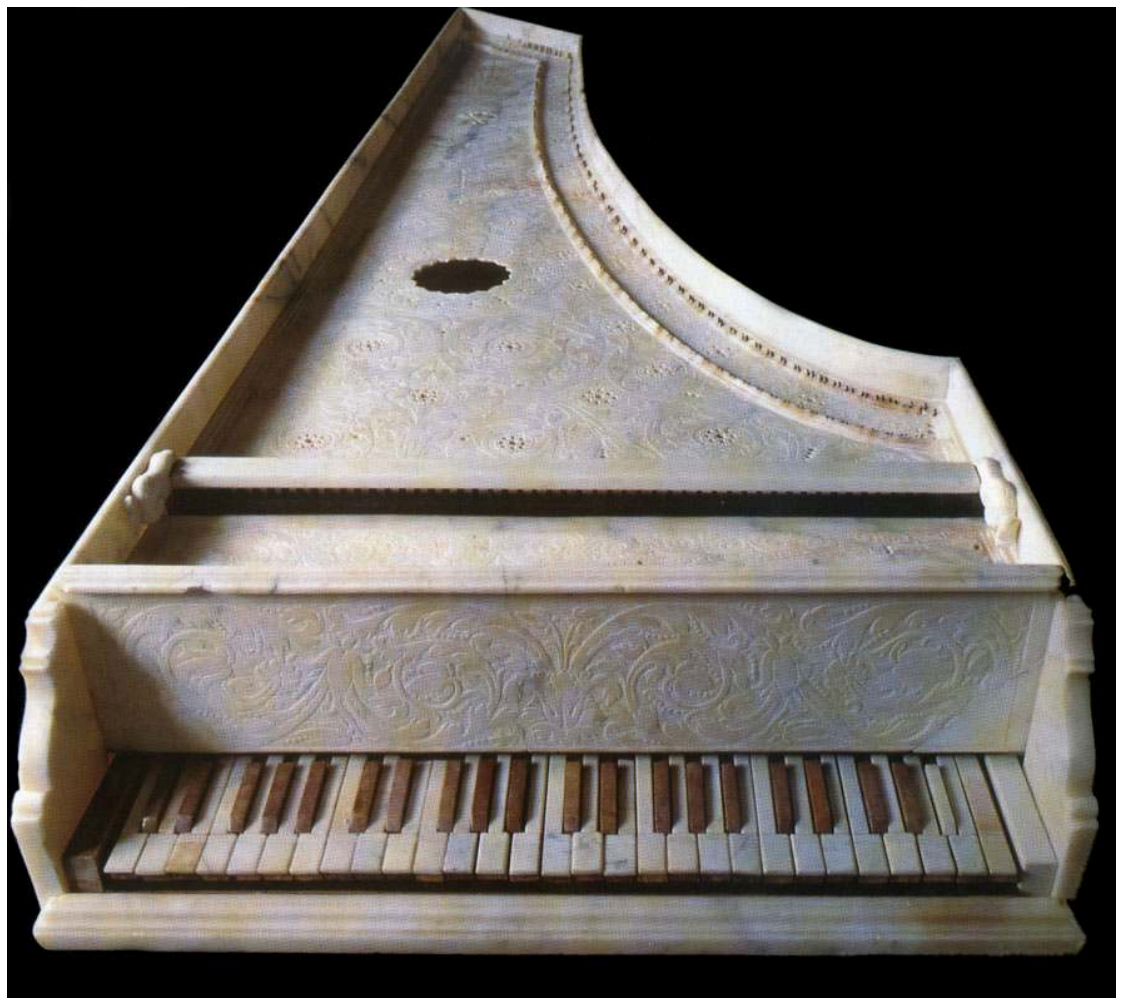

Clavecin en marbre de Carrare pour le duc Francesco II d'Este, Michele Antonio Grandi, 1687. Modène, Galleria Estense.

Crédit : Bernardini 2005 : 102. 
Une autre étape essentielle dans l'histoire des collections d'outils et d'instruments est marquée au XIX ${ }^{\mathrm{e}}$ siècle par la naissance des musées d'art décoratifs et d'ethnographie. L'intérêt de l'époque moderne pour les techniques avait continué de se développer avec la Révolution industrielle. Déjà, l'Encyclopédie ou Dictionnaire raisonné des sciences, des arts et des métiers publiée entre 1751 et 1772 avait eu l'ambition de rendre compte autant que possible de l'ensemble des «sciences, arts et métiers " en décrivant au moyen de gravures détaillées des centaines d'outils, d'instruments et de machines ${ }^{11}$. Précipitant les innovations, cette démonstration du savoir-faire humain trouva son prolongement dans la collection didactique du Conservatoire des arts et métiers de Paris, fondé en 1794. S'ensuivront les nombreuses présentations des métiers et de l'industrie des expositions universelles $\mathrm{du} \mathrm{XIX}^{\mathrm{e}}$ et $\mathrm{du}$ début $\mathrm{du} \mathrm{XX}^{\mathrm{e}}$ siècle, qui furent de grandes expositions d'objets. La première d'entre elles, organisée à Londres en 1851, fut à l'origine du South Kensington Museum, futur Victoria \& Albert Museum, qui servit à son tour de modèle à d'autres musées dits d'«arts décoratifs". Ces derniers se concevaient aussi comme des musées de techniques ${ }^{12}$. Tournés vers l'avenir, ils entendaient montrer toutes sortes d'artefacts caractéristiques issus de siècles révolus ou de régions lointaines et qui témoignaient d'une grande virtuosité : dans cette nouvelle ère industrielle, ils semblaient dignes d'être imités et généraient un esprit d'émulation, notamment pour le commerce. Quant aux musées d'ethnographie, ils étaient plus portés sur les productions du quotidien rural et artisanal et suscitaient un intérêt renforcé par les effets cumulés de l'industrialisation, de l'urbanisation et de la colonisation.

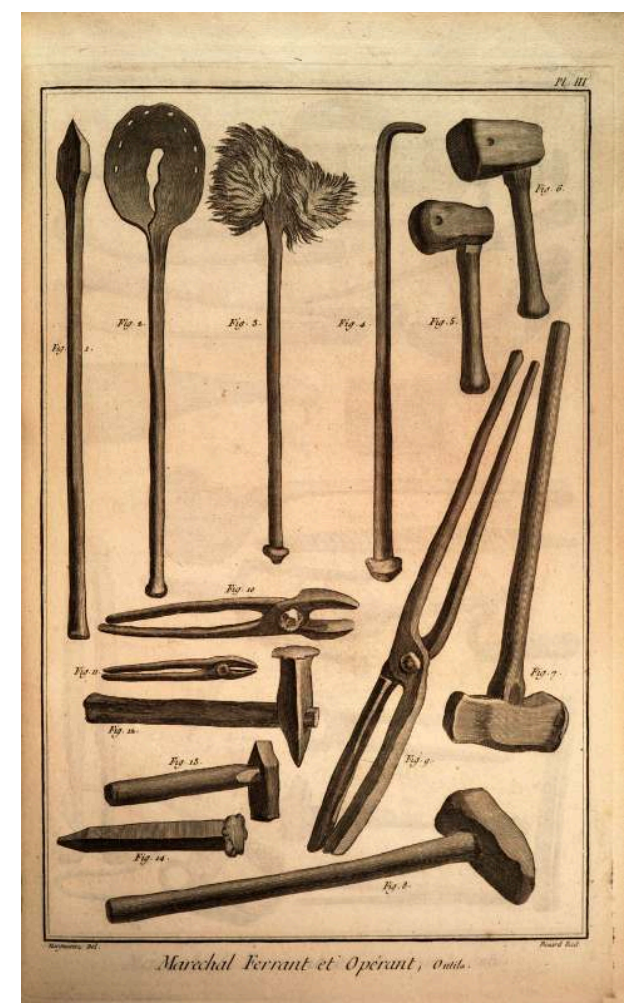

Diderot \& D'Alembert 1751-1772, Planches, t. 7, « Maréchal Ferrant », pl. III.

Bayerische Staatsbibliothek München, 24061192 Enc. 9, A-6, urn :nbn :de :bvb :12-bsb10325923-8

5 C'est dans ce contexte que se diffusa le terme d' " objet d'art " ${ }^{13}$. En allemand, on employa beaucoup le mot Gerät ${ }^{14}$ pour désigner de manière générale les objets 
fabriqués et utilisés. Gerät renvoyait moins à l'esthétique qu'à l'importance de ces objets dans les phénomènes de civilisation. Selon cette acception, certains historiens de l'art s'efforcèrent d'appréhender l'histoire de l'ensemble des artefacts humains. Gottfried Semper, lui-même impliqué dans la réforme anglaise des arts décoratifs et dans la question des musées, développa à partir du concept "d'esthétique pratique " une théorie du style qui trouvait son point de départ dans une classification des matières et de leur mise en œuvre ${ }^{15}$. Cette articulation entre les fonctions et les formes favorisa également l'expression " art populaire » : Alois Riegl, longtemps conservateur au Musée autrichien de l'art et de l'industrie, considérait ce type d'objets du point de vue des conditions sociales de leur production. Il formula plus tard sa notion de Kunstwollen ou « vouloir artistique » qui entendait rendre compte des formes de tous les types d'objets d'une même époque ${ }^{16}$. Aby Warburg développa pour sa part une conception de Gerät permettant de décrire toutes les productions matérielles humaines comme des satisfactions de besoins à la fois fonctionnels et esthétiques ${ }^{17}$. Henri Focillon prit à son tour parti pour une étude des humains fondée sur tous leurs objets ${ }^{18}$, et George Kubler s'efforça à sa suite de décrire les rapports morphologiques observables entre tous les artefacts possibles ${ }^{19}$.

\section{Processus}

Parallèlement à ces activités de collection et de mise en exposition, et à cet intérêt pour les formes des objets fonctionnels, un autre courant s'était développé qui entendait étudier l'histoire des outils et des instruments de façon technologique, c'est-à-dire en considérant l'acte même de leur utilisation. La découverte d'outils de silex " antédiluviens" par Jacques Boucher de Perthes, vers le milieu du XIX siècle ${ }^{20}$, révolutionna non seulement les connaissances sur l'origine des humains mais détermina aussi l'étude scientifique des techniques, les observations des préhistoriens concordant avec celles des ethnographes et, plus tard, des primatologues. S'appuyant sur la théorie de l'évolution de Charles Darwin, le lieutenant général Augustus Pitt Rivers organisa sa vaste collection d'artefacts ethnographiques et archéologiques selon des types fonctionnels et dans un ordre chronologique, avant de la transférer en 1884 à Oxford dans un musée qui porte son nom ${ }^{21}$. Le philosophe allemand Ernst Kapp publia en 1877 ses Principes d'une philosophie de la technique où il formulait la théorie de la "projection organique» selon laquelle les outils prolongeraient les membres humains ${ }^{22}$. En 1935, un article du sociologue et ethnologue français Marcel Mauss sur les "techniques du corps" donna une impulsion décisive au développement des recherches empiriques ${ }^{23}$. L'ethnologue et préhistorien André Leroi-Gourhan présenta ensuite plusieurs études novatrices sur l'anthropologie des techniques ${ }^{24}$, selon une approche développée jusqu'à aujourd'hui sous le nom de technologie culturelle ${ }^{25}$. Des réflexions apparentées ont également été conduites dans le domaine récent de la sociologie des sciences et des techniques ${ }^{26}$, dans des travaux empiriques et théoriques sur la culture matérielle ${ }^{27}$, dans des études de philosophie pragmatique ${ }^{28}$ ou encore dans le champ des sciences politiques ${ }^{29}$. En approfondissant les questions de l'évaluation et de l'appréciation éthique, la philosophie des techniques ${ }^{30}$ prend aujourd'hui les caractéristiques d'une science appliquée, qui joue un rôle important dans certaines universités ${ }^{31}$. De plus en plus, les techniques occupent le centre des préoccupations de nos sociétés. 
Ce survol bref et sûrement partiel suffit pour constater à quel point les recherches en sciences sociales et les débats publics sur les processus techniques se sont développés loin de l'histoire de l'art. Tant celle-ci, qui s'est vouée d'abord et surtout à la tradition artistique occidentale, que l'anthropologie de l'art, développée ensuite à partir de terrains extra-européens ${ }^{32}$, se sont souvent conformées à la conception de l'art dominante en Europe depuis l'époque moderne et ont ainsi étudié avant tout des phénomènes esthétiques. Elles ont donc négligé le domaine de la technique, alors même que le mot latin ars s'apparente largement au terme grec technè, ou encore qu'en allemand, l'étymologie qui associe " Kunst » au savoir-faire (« können ») souligne aussi un lien étroit avec le domaine des techniques. Ce n'est pourtant que vers le milieu du $\mathrm{XVIII}^{\mathrm{e}}$ siècle que l'on distingua, dans le champ de l'art, entre les domaines de la technique et de l'esthétique ${ }^{33}$. De cette situation résulte un défi : les catégories de l'art et de la technique, constitutives de différentes disciplines académiques, doivent être historicisées de manière à pouvoir surmonter des divisions conceptuelles qui sont récentes et spécifiques : elles relèvent finalement plus de l'idéalisme occidental du xix ${ }^{e}$ siècle qu'elles ne peuvent servir les sciences sociales dans les tâches qui sont aujourd'hui les leurs. Au-delà de l'interdisciplinarité, nécessairement conditionnée par des conceptions anciennes et clivantes, il s'agit d'ouvrir de nouveaux champs de recherche métadisciplinaires, plus ouverts car tenant explicitement compte de l'histoire des disciplines ${ }^{34}$.

7 Plusieurs pas ont déjà été faits dans cette direction. L'historien de l'art Pierre Francastel a développé une sociologie de l'art qui accorde une place centrale au développement technique ${ }^{35}$. La technologie culturelle française a inspiré des travaux historiques qui portent aussi sur des objets de l'histoire de l'art, comme la construction architecturale ${ }^{36}$. Dans son livre Art and Agency, l'anthropologue Alfred Gell a proposé ce qui peut être considéré comme une analyse de techniques sociales incluant un large spectre d'objets ${ }^{37}$. Hans Belting a plaidé pour une anthropologie qui envisage les images dans une relation physique (soit en fait technique) aux humains ${ }^{38}$. D'autres ont recouru au concept de "Kulturtechnik » ou " technique culturelle » pour souligner que les images, l'écriture et les chiffres, constitutifs des cultures au sens intellectuel du terme, doivent être considérés comme des techniques du symbolique ${ }^{39}$. Mais une réserve doit ici être apportée, car toute technique est à la fois matérielle et symbolique, et que ceci détermine précisément ce qu'est la culture. Cultura, en latin, désignait à la fois les techniques intellectuelles et agricoles. Le concept de «Kulturtechnik » risque donc de perpétuer la distinction entre technique et culture qu'il voudrait dépasser, et il serait plus habile de reconnaître que les deux termes sont étroitement liés et au fond synonymes. Ils renvoient tous deux aux expériences à la fois opératoires et cognitives des humains dans leur environnement - l'expression « technologie culturelle » (soit en allemand «Kulturwissenschaftliche Technikforschung») est en ce sens plus pertinente ${ }^{40}$.

8 Ainsi conçue, la technique offre une perspective pour une réflexion qui ne veut plus choisir ses champs de recherche entre un domaine matériel tirant du côté des sciences naturelles et un domaine cognitif se concentrant sur les symboles - mais qui, bien plutôt, a pour ambition d'éclairer les formes variées de la vie sociale humaine en dépassant ce type de distinction ${ }^{41}$. Les travaux récents qui surmontent la différence entre anthropologie des techniques et anthropologie de l'art constituent à cet égard une avancée décisive, en cessant de désarticuler en théorie les objets, entre technique 
et esthétique, pour les saisir au contraire dans leur globalité, c'est-à-dire en relation avec des processus à la fois matériels et symboliques. ${ }^{42}$

\section{Représentations}

L'histoire de l'art devrait-elle, sur cette lancée, se fondre dans une vaste anthropologie historique des techniques ${ }^{43}$ ? Pas exactement, car si elle peut contribuer significativement à ce champ de recherche c'est aussi par son histoire et par son expertise propres. Sont ici en jeu le concept occidental et moderne de l'« art», et la question souvent débattue des relations entre techniques et vie sociale : les techniques donnent-elles forme à des relations sociales préexistantes, ou en façonnent-elles de nouvelles et comment ${ }^{44}$ ? Le point crucial est que les processus techniques, par-delà leur mise en œuvre, sont représentés non seulement par des mots et des récits ${ }^{45}$ ou des chiffres, mais aussi notamment par des images mentales et matérielles. Technè dans l'Antiquité, tout comme ars, ingenium ou encore industria au Moyen Âge, signifient aussi la ruse. Ces termes suggèrent la maîtrise de processus ambivalents, dont la fascination est capturée et rendue dans une autre élaboration.

9 Ainsi les techniques vont-elles de pair avec des représentations, lesquelles participent pleinement de - et à - leur socialisation. Une étude ethnographique, par exemple, a mis en évidence que l'imaginaire contemporain des techniques de traitement des déchets relève de guerres de titans, de feux infernaux et de forges d'alchimistes ${ }^{46}$ : des représentations anciennes, liées à d'autres techniques et à des mythes, peuvent être mobilisées pour se saisir de pratiques actuelles et en parler. Des «Leitbilder» ou « images directives ", qui fondent des modèles culturels tels que l'idée de croissance ou celle de durabilité, jouent un rôle décisif dans les innovations techniques ${ }^{47}$. Celles-ci feront à leur tour l'objet d'interprétations symboliques, littéraires par exemple : c'est ainsi que la science-fiction est peuplée de machines douées de vie et de créatures génétiquement modifiées ${ }^{48}$. Dans cette situation, l'histoire de l'art peut mettre à profit son savoir-faire pour analyser les représentations matérielles d'objets et de processus techniques, afin de comprendre leurs enjeux sociaux. L'étude de trois images d'outils ou d'instruments évoquant des gestes techniques, destinées à faire évoluer les relations sociales dans des situations historiques, techniques et artistiques diverses entre le $\mathrm{xv}^{\mathrm{e}}$ et le $\mathrm{xx}^{\mathrm{e}}$ siècle, permettra de montrer comment.

La basilique de San Miniato al Monte à Florence abrite dans son transept sud une fresque du début du Xve siècle figurant le Christ ressuscité, debout, tenant la croix de sa main droite tandis que de la gauche il indique sa plaie au flanc. Le Christ est entouré de près de vingt-cinq outils dont les pointes et les tranchants sont dirigés vers son corps dévêtu. Son corps et la croix ont été peints a fresco, directement sur l'enduit frais, mais les outils ont été ajoutés plus tard, a secco et au moyen de feuilles de métal figurant les parties d'acier. Les outils sont ainsi des corps étrangers, tant selon le processus de fabrication de l'image que dans sa logique visuelle et narrative. Bien que l'on ne distingue plus dans la plupart des cas que les contours des applications métalliques aujourd'hui disparues, on imagine ce qu'a dû être leur présence brillante et menaçante, sur un fond jadis entièrement bleu. Sous l'image, une inscription facile à lire, en langue vernaculaire et que sa rime fait mémoriser, met en garde : « chi no guarda la domenica sca et a xpo no a devotione dio gli dara la eterna danatione ", " celui qui ne respecte pas le saint dimanche et ne vénère pas le Christ, Dieu le damnera pour l'éternité ». Pour 
éviter cette condamnation, chacun est exhorté à laisser reposer ses outils le dimanche : manier un outil ce jour-là réitérerait le martyr du Sauveur, tel que le type iconographique apparenté des Arma Christi le commémorait. L'image était clairement destinée à marquer une communauté, d'une part en prônant l'interruption des activités techniques au profit d'un rassemblement dans l'église pour la célébration de la messe et de l'Eucharistie, et d'autre part par le choix des outils représentés, qui font référence à différents corps de métiers comme ceux des tailleurs, des bouchers, des charpentiers, etc.

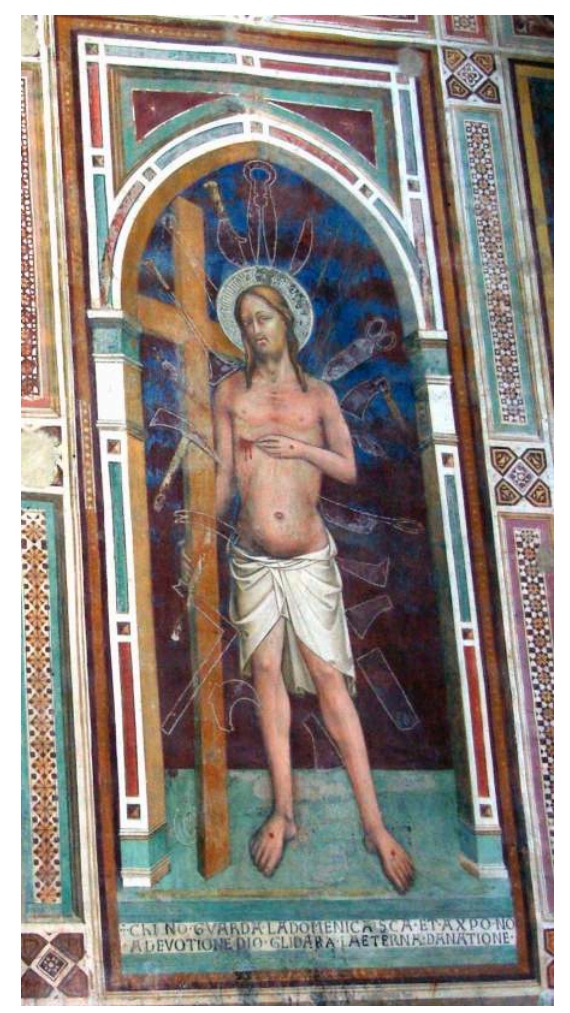

Christ du dimanche, fresque, Florence, San Miniato al Monte, Mariotto di Cristofano, début du xve siècle.

Cliché : Francis Fletcher.

11 Cette image a été peinte sur le seul pan de mur de la basilique depuis lequel, lorsque le portail principal est ouvert, le regard porte jusqu'en bas de la colline sur la cathédrale Santa Maria del Fiore de Florence, et surtout sur sa fameuse coupole qui était alors en construction. Ce ne saurait être un hasard, car on a précisément travaillé certains jours fériés sur ce chantier exceptionnel, par exemple pour maintenir l'humidité des murs, comme des mentions d'archives l'indiquent pour les étés 1427 et $1428{ }^{49}$. La règle du repos dominical a donc été rompue au cœur de la cité florentine, au profit d'une réalisation techniquement innovante, ce à quoi les moines de San Miniato, dominant la ville, répondirent manifestement par cette fresque. De tels «Christ du dimanche » sont surtout fréquents dans les paroisses rurales des Alpes, et parmi tous les exemples connus, c'est celui-ci dont l'inscription formule la mise en garde la plus sévère ${ }^{50}$. La représentation de grands ciseaux au-dessus de la tête du Christ s'adresse probablement d'une façon très concrète et explicite à la corporation des tisserands de laine : la commune de Florence lui ayant confié la construction de la cathédrale, c'est elle qui portait la responsabilité de ce travail dominical jugé abusif. 
Une autre mise en image, bien différente, d'une relation idéale entre technique et société est offerte par les figures d'un ouvrier et d'une kolkhozienne conçues par Vera Mukhina pour le pavillon soviétique de l'Exposition universelle organisée à Paris sous le titre Exposition Internationale des Arts et des Techniques dans la vie moderne en $1937{ }^{51}$. L'importance de cette représentation est déjà mise en évidence par les proportions relatives du bâtiment et des figures : la tour de la façade devant la halle d'exposition s'étirant vers l'arrière était haute de trente-cinq mètres, et la sculpture à laquelle elle servait de socle, de vingt mètres. L'ensemble culmine dans une paire d'outils, le marteau et la faucille, emblèmes de l'Union Soviétique, brandis triomphalement par les deux personnages de sorte que leurs silhouettes se détachent sur le ciel et forment une figure héraldique étincelante. Cette double sculpture est en effet constituée de plaques d'un alliage inoxydable d'acier et de nickel, épaisses de quelques millimètres, soudées et rivetées les unes aux autres comme il était alors courant en aéronautique. L'œuvre s'affirmait ainsi comme une entreprise collective, relevant tout à la fois de l'art, de l'architecture et de l'industrie, et présentait un modèle de société déterminé par l'harmonie existant entre ces activités. Or, avec le marteau et plus encore la faucille, on représentait des outils que l'industrialisation rendait précisément caducs, tandis que les figures se rattachaient à l'Antiquité grecque en variant sur le type iconographique de l'allégorie de la Victoire. Cette image du travail manuel en célébrait donc la disparition, et formulait en tant qu'œuvre d'art industriel une utopie technico-sociale.

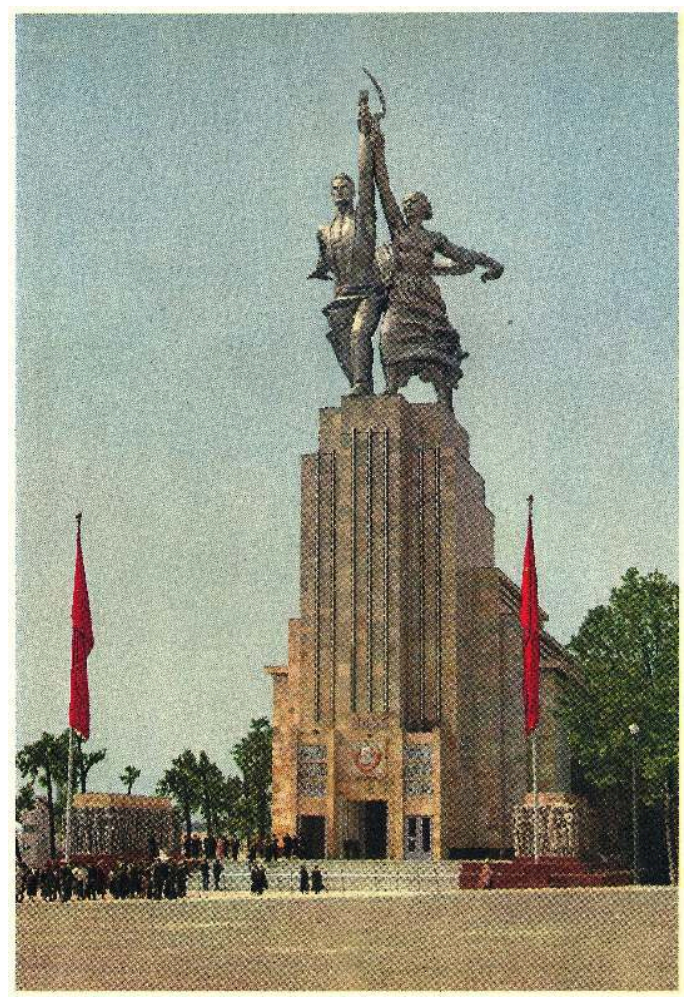

Vera Mukhina, sculpture pour le pavillon soviétique de l'« Exposition Internationale des Arts et Techniques dans la Vie Moderne » à Paris en 1937.

Cliché : Swift 2007 : 182 


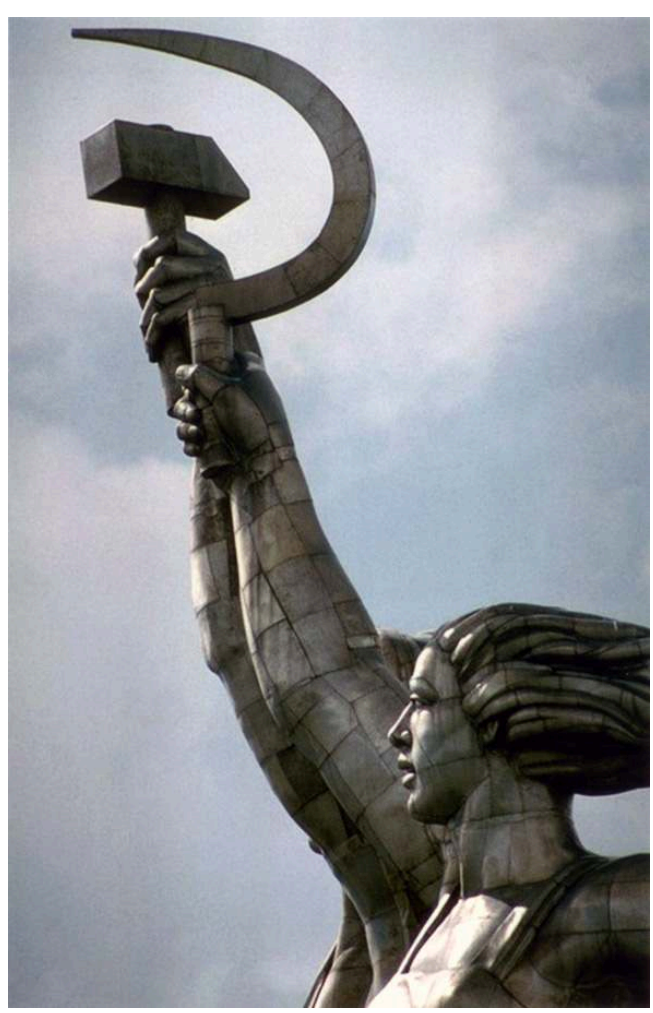

Vera Mukhina, sculpture pour le pavillon soviétique de l'« Exposition Internationale des Arts et Techniques dans la Vie Moderne » à Paris en 1937, détail.

Cliché : Werckmeister 2002: 47.

L'« iconographie des matériaux » développée par l'histoire de l'art germanophone s'est concentrée avant tout sur des aspects sémantiques ${ }^{52}$. Elle apparaît particulièrement adaptée à l'analyse des représentations techniques, à condition de la compléter par une « iconologie de la technique » qui considérera en outre la mise scène des processus. L'histoire de l'art se trouve ainsi incitée à interroger l'histoire riche et complexe des rapports entre technique et art, et entre autres le statut de l'historiographie de l'art en tant que narration spécifique, mais déterminante, dans l'histoire occidentale des techniques ${ }^{53}$. Ceci renvoie en particulier à la « Renaissance » florentine, lorsque furent rédigées des biographies d'artistes dont l'influence fut très grande et lorsqu'apparurent des théories sur l'art qui déclassaient les aspects techniques et matériels.

Le peintre Federico Zuccari fit ainsi construire à partir de 1578, pour son nouvel atelier de Florence, une façade à la fois spectaculaire et programmatique, qui comprend trois reliefs sculptés représentant divers outils et instruments comme s'ils avaient été pris dans la pierre. Complètement dégagés du mur, ces objets auraient été pour ainsi dire expulsés de l'atelier. Cette allusion au non-finito de Michel Ange, mort en 1564, servait probablement de contrepoint au concept du disegno, puisque l'on peut supposer que le grand cadre de pierre de l'étage supérieur, légèrement incliné vers le spectateur à la manière d'une toile et vide aujourd'hui, était destiné à accueillir une allégorie du dessin ${ }^{54}$. L'art du dessin était en effet défini et célébré par Zuccari comme primant sur toute autre technique, comme une "métatechnique " ${ }^{55}$ censée reposer sur la traduction immédiate et virtuose d'images mentales, plutôt que sur une pratique routinière. Le contraste entre les blocs de pierre du registre inférieur de la façade, bruts ou lisses, et les briques de la partie haute renvoie en ce sens aussi bien à l'architecture des palais romains de l'époque, alors inhabituelle à Florence, qu'à la 
maîtrise de la natura par l'ars. Première en son genre, cette façade d'atelier était ostensiblement détachée de la maison d'habitation, qui avait appartenu précédemment au peintre Andrea del Sarto (1486-1530), célébré à Florence, dont Zuccari prenait ainsi la suite. Les reliefs décorés d'outils, quant à eux, semblent emprunter aux métopes de la cour intérieure d'une maison spectaculaire que le sculpteur Leone Leoni s'était faite construire à Milan quelques années auparavant ${ }^{56}$. Une telle ambition poursuivait les réflexions de Giorgio Vasari, l'auteur en 1550 des Vies des meilleurs peintres, sculpteurs et architectes ${ }^{57}$, qui lui aussi avait décoré ses propres maisons, à Arezzo et Florence, de fresques figurant des théories artistiques. Zuccari, surtout, avait alors repris le chantier prestigieux, commencé par Vasari, des peintures intérieures de la coupole de la cathédrale de Florence. Mais aussi publique qu'ait été la façade de son atelier, ce discours concernait avant tout l'artiste lui-même et un cercle étroit et cultivé de connaisseurs, de commanditaires, et de collègues tels qu'ils se réunissaient par exemple à l'Accademia del Disegno fondée en 1563. La différenciation entre " technique » et " art » révèle ici son aspect social: explicitement séparés des artisans, les "artistes " ne constituaient qu'un très petit groupe d'individus. Ils en étaient d'autant plus flexibles et innovants. Ceci fut particulièrement évident à Florence dès le $\mathrm{xv}^{\mathrm{e}}$ siècle, dans un moment d'émulation dont l'histoire de l'art s'efforce de rendre compte ${ }^{58}$.

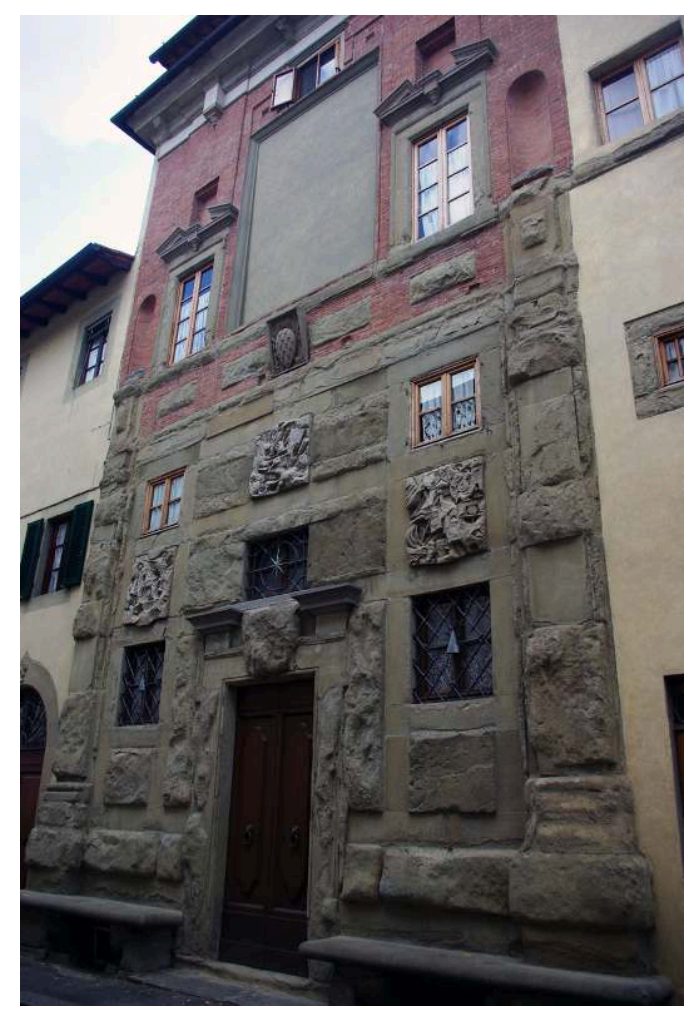

Façade de l'atelier de Federico Zuccari, Florence, 1575-1579.

Cliché : Philippe Cordez. 


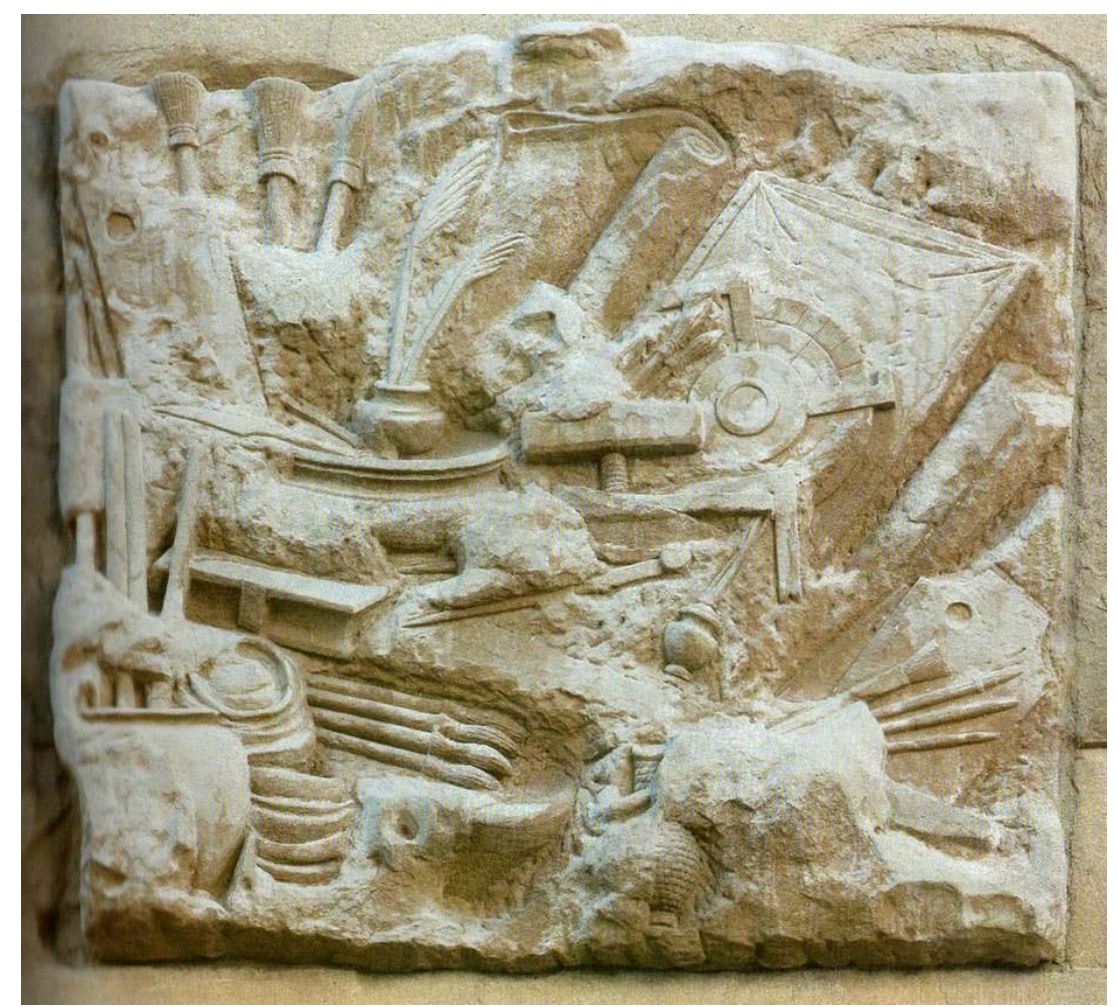

Relief supérieur de la façade de l'atelier de Federico Zuccari.

Cliché : Acidini 2009 : 167.

Montrer des œuvres d'art impliquait alors toujours la société dans son entier, mais désormais bien plus par différentes techniques d'appropriation que par une familiarité directe de nombreuses personnes avec les processus de fabrication, comme cela était encore le cas pour les objets plus communs. La production artistique spécialisée eut ainsi pour conséquence un déplacement du rapport entre les techniques et leurs représentations. Le même constat peut être fait, plus généralement pour les marchandises relevant des "arts décoratifs", au sens du XIXe siècle, ou de l'industrie. Dans les sociétés où priment des techniques qui évoluent peu et que la plupart comprennent, celles-ci déterminent pour une large part les représentations qui fondent la vie sociale. Dans le contexte de l'art et de l'industrie, au contraire, la production symbolique de la vie en société est moins exactement liée à des techniques de fabrication, devenues plus complexes et moins bien connues, et recourt davantage à des objets plus élaborés et sans cesse renouvelés que la majorité s'approprie en les admirant. Ces objets raffinés s'imposent alors comme le support des représentations de la société, et conditionnent la vie sociale par leur publicité, leur propriété ou leur usage ${ }^{59}$.

Les techniques et leurs représentations se déterminent mutuellement, et déterminent ensemble les sociétés. Il apparaît donc logique que la différenciation sémantique entre outil et instrument, qui entend opérer une distinction entre le travail et l'art ou les sciences, ait été particulièrement d'actualité alors que les productions industrielles se développaient dans le monde occidental, remettant en question la pertinence des représentations techniques traditionnelles. Le développement technique a été de pair avec une réorganisation symbolique, en un processus qui n'a fait que s'intensifier et se compliquer depuis. Pour l'histoire de l'art, les mots «outil» et «instrument» constituent ainsi une incitation à considérer les techniques à partir des objets, des 
processus à l'œuvre et de leurs représentations. Il ne s'agit pas simplement d'observer l'utilisation de techniques artisanales, physiques ou chimiques, digitales ou même biologiques dans la production d'œuvres d'art: il en va bien plutôt, par une problématisation plus poussée et par une mise en situation historique de la notion d'art, et notamment par un rapprochement productif avec l'anthropologie des techniques, de la participation de l'histoire de l'art à l'entreprise collective des sciences sociales.

\section{BIBLIOGRAPHY}

Acidini, C. 2009 « Un episodio fiorentino : le formelle dello Studio Zuccari nella facciatamanifesto ", in C. Acidini \& E. Capretti dir. Innocente e calunniato. Federico Zuccari (1539/40-1609) e le vendette d'artista. Florence : Giunti : 164-171.

Akrich, M. 1994 « Comment sortir de la dichotomie technique/société. Présentation des diverses sociologies de la technique », in B. Latour \& P. Lemonnier dir. De la préhistoire aux missiles balistiques. L'intelligence sociale des techniques. Paris : La Découverte : 105-131.

Audouze F. \& N. Schlanger dir. 2004 Autour de l'homme. Contexte et actualité d'André Leroi-Gourhan. Antibes : Éditions APDCA.

Balfet, H. dir. 1991 Observer l'action technique. Des chaînes opératoires pour quoi faire? Paris : Éditions du CNRS.

Baudrillard, J. 1968 Le système des objets. Paris : Gallimard.

Becker, C. \& J. H. von Hefner-Alteneck dir. 1852-1863 Kunstwerke und Geräthschaften des Mittelalters und der Renaissance, 3 vol. Francfort sur le Main : Keller.

Belting, H. 2004 [éd. all. 2001] Pour une anthropologie des images. Paris : Gallimard.

Bernardini, M. G. dir. 2005 Un cembalo in marmo per Francesco II d'Este. Modène : Fondazione Cassa di Risparmio.

Bessac, J.-C. 1986 L'outillage traditionnel du tailleur de pierre de l'Antiquité à nos jours. Paris : Éditions du CNRS.

Boucher de Perthes, J. 1847-1864 Antiquités celtiques et antédiluviennes. Mémoire sur l'industrie primitive et les arts à leur origine. Paris : Treuttel et Wurtz.

Braunfels, W. 1961 « Die moderne Kunst und der technische Fortschritt », Jahrbuch für Ästhetik und allgemeine Kunstwissenschaft $6: 17-43$.

- 1965 « Drei Bemerkungen zur Geschichte und Konstruktion der Florentiner Domkuppel », Mitteilungen des Kunsthistorischen Institutes in Florenz 11/4 : 203-226.

- 1979 [1953] Mittelalterliche Stadtbaukunst in der Toskana. Berlin : Mann.

Bredekamp, H., M. Bruhn \& G. Werner dir. 2003 Bildwelten des Wissens. Kunsthistorisches Jahrbuch für Bildkritik. Berlin : Akademie-Verlag. 
Bredekamp, H., B. Schneider \& V. Dünkel dir. 2015 [éd. all. 2008] The Technical Image. A History of Styles in Scientific Imagery. Chicago : University of Chicago Press.

Brilli, E., P.-O. Dittmar \& B. Dufal dir. 2010 L'Atelier du Centre de recherches historiques. Revue électronique du CRH 6, Faire l'anthropologie historique du Moyen Âge. [En ligne] : journals.openedition.org/acrh/1911.

Coleu, M. \& M. Pardo dir. 2005 Inventions of the Studio. Renaissance to Romanticism, Chapell Hill \& Londres : University of North Carolina Press.

Conforti, M. 1997 «Les musées des arts appliqués et l'histoire de l'art », in E. Pommier dir. Histoire de l'histoire de l'art, Paris : Klincksieck : 327-347.

Cordez, P. 2012 « Werkzeuge und Instrumente in Kunstgeschichte und Technikanthropologie », in P. Cordez \& M. Krüger dir. Werkzeuge und Instrumente, Berlin : Akademie Verlag (« Hamburger Forschungen zur Kunstgeschichte $8 »)$.

Crawford, M. B. 2010 [éd. angl. 2009] Éloge du carburateur. Essai sur le sens et la valeur du travail, Paris : La Découverte.

Cresswell, R. 1994 « La nature cyclique des relations entre le technique et le social. Approche technologique de la chaîne opératoire ", in B. Latour \& P. Lemonnier dir. De la préhistoire aux missiles balistiques. L'intelligence sociale des techniques, Paris : La Découverte : 275-288.

- 1996 Prométhée ou Pandore ? Propos de technologie culturelle, Paris.

- 2003 « Geste technique, fait social total. Le technique est-il dans le social ou face à lui ? ", in G. Guille-Escuret dir. Techniques \& Culture 40, Efficacité technique, efficacité sociale : 125-152.

David, J. 1997 L'outil, Turnhout : Brepols (« Typologie des sources du Moyen Âge occidental 78 »).

Descola, P. 2005 Par-delà nature et culture, Paris : Gallimard.

- dir. 2010 La Fabrique des images. Visions du monde et formes de la représentation, Paris : Somogy.

Diderot, D. \& J. B. le Rond D’Alembert dir. 1751-1772 Encyclopédie ou Dictionnaire raisonné des sciences, des arts et des métiers, 28 vol., Paris.

Diers, M. \& M. Wagner dir. 2010 Topos Atelier. Werkstatt und Wissensform, Berlin : Akademie-Verlag (« Hamburger Forschungen zur Kunstgeschichte 7 »).

D’Onofrio, S. 2005 « Chaque chose a son "galbe". Style, technique et esthétique à propos d'outils siciliens ", in B. Martinelli dir. L'interrogation du style. Anthropologie, technique et esthétique, Aix en Provence : Aix en Provence : Publications de l'Université de Provence : 87-96.

D’Onofrio, S. \& F. Joulian dir. 2006 Dire le savoir-faire. Gestes, techniques et objets, Paris : L'Herne («Cahiers d'anthropologie sociale $1 »$ ).

Ducci, A. 2006 « Henri Focillon, l'arte popolare e le scienze sociali », Annali di critica d'arte 2 : 341-389.

Dufrêne, T. \& A.-C. Taylor dir. 2009 Histoire de l'art et anthropologie. [En ligne], mis en ligne en juillet 2009. URL : actesbranly.revues.org/60. Consulté le 20 décembre 2017. Certains textes sont imprimés sous le titre Cannibalismes disciplinaires. Quand l'histoire de l'art et l'anthropologie se rencontrent, Paris : Musée du quai Branly).

Flichy, P. 2001 L'imaginaire d'Internet, Paris.

Focillon, H. 2013 [1934] La vie des formes, Paris : Presses Universitaires de France.

Francastel, P. 1988 [1956] Art et technique aux XIX et XX siècles, Paris : Gallimard. 
- 1989 [1970] Études de sociologie de l'art, Paris : Gallimard.

Gell, A. 2009 [éd. angl. 1998] L'art et ses agents. Une théorie anthropologique, Dijon : Les presses du réel

Gétreau, F. dir. 2006-2007 Musique. Images. Instruments. Revue française d'organologie et d'iconographie musicale 8-9, Les collections d'instruments de musique.

Goody, J. 2003 [éd. angl. 1997] La peur des représentations. L'ambivalence à l'égard des images, du théâtre, de la fiction, des reliques et de la sexualité, Paris : La Découverte.

Grimm J. et al. 1854-1960 Deutsches Wörterbuch, 32 vol., Leipzig : Hirzel.

Grob B. \& H. Hooijmaijers dir. 2006 Who Needs Scientific Instruments? Conference on Scientific Instruments and their Users, Leyde : Museum Boerhaave.

Guchet, X. 2008 « Évolution technique et objectivité technique chez Leroi-Gourhan et Simondon », Revue Appareil 2. [En ligne], mis en ligne le 11 septembre 2008. URL : journals.openedition.org/appareil/580. Consulté le 20 décembre 2017.

Guille-Escuret, G. dir. 2003 Techniques \& Culture 40, Efficacité technique, efficacité sociale.

Haines, M. 2002 The Years of the Cupola : Digital archive of the sources of the Opera di Santa Maria del Fiore, 1417-1436. [En ligne], mis à jour en août 2005. URL : duomo.mpiwg-berlin.mpg.de. Consulté le 20 décembre 2017.

Heidelberger, M. 2006 « Experiment und Instrument », in H. Schramm, L. Schwarte \& J. Lazardzig dir. Instrumente in Kunst und Wissenschaft. Zur Architektonik kultureller Grenzen im 17. Jahrhundert, Berlin : De Gruyter : 378-397.

Hengartner, T. dir. 2007- Kulturwissenschaftliche Technikforschung, 8 vol. parus, Zurich : Chronos.

Holländer, B. 2000 « Technik und Arbeit in den Tafelbänder der Encyclopédie », in H. Holländer dir. Erkenntnis, Erfindung, Konstruktion. Studien zur Bildgeschichte von Naturwissenschaften und Technik vom 16. bis zum 19. Jahrhundert, Berlin : Mann : 789-806.

Hubig, C., A. Huning \& G. Ropohl dir. 2013 [2000] Nachdenken über Technik. Die Klassiker der Technikphilosophie und neuere Entwicklungen, Berlin : edition sigma.

Irrgang, B. 2001-2002 Philosophie der Technik, 1 Technische Kultur. Instrumentelles Verstehen und technisches Handeln, 2 Technische Praxis. Gestaltungsperspektiven technischer Entwicklung, 3 Technischer Fortschritt. Legitimitätsprobleme innovativer Technik, Paderborn etc. : Schöningh. Jamard, J.-L. dir. 2004 Techniques \& Culture 43-44, Mythes. L'origine des manières de faire. Julien, M.-P. \& C. Rosselin dir. 2005 La culture matérielle, Paris : La Découverte. - dir. 2009 Le sujet contre les objets... tout contre. Ethnographies de cultures matérielles, Paris : CTHS. Julien, M.-P. \& J.-P. Warnier dir. 1999 Approches de la culture matérielle. Corps à corps avec l'objet, Paris : L'Harmattan.

Jungen, B. 2005 Kunstpolitik versus Kunst. Leben und Werk der Bildhauerin Vera Muchina (1889-1953), Bielefeld : Kerber.

Kapp, E. 2007 [éd. all. 1877] Principes d’une philosophie de la technique, Paris : Vrin.

Karafyllis, N. C. 2000 Nachwachsende Rohstoffe - Technikbewertung zwischen den Leitbildern Wachstum und Nachhaltigkeit, Opladen : Leske + Budrich.

- 2004 « Zum Systemverständnis von Leitbildern in der Technikentwicklung und -gestaltung », in K. Kornwachs dir. Technik - System - Verantwortung, Münster etc. : LIT Verlag : 485-498. 
Korey, M. 2002/2003 « Die Dresdner Kunstkammer. Instrumente des Wissens und des Könnens ", Jahrbuch der Staatlichen Kunstsammlungen Dresden 30 : 47-60.

Korff, G. 2003 « Kulturforschung im Souterrain. Aby Warburg und die Volkskunde », in K. Maase $\&$ B. Warneken dir. Unterwelten der Kultur. Themen und Theorien der volkskundlichen Kulturwissenschaft, Cologne, Weimar \& Vienne : Böhlau : 143-178.

- 2012 « Schmerzlose Körperteile. Zu Aby Warburgs Anthropologie des “Geräts” » in P. Cordez \& M. Krüger dir. Werkzeuge und Instrumente, Berlin : Akademie Verlag, 2012 (« Hamburger Forschungen zur Kunstgeschichte 8 ») : 129-149.

Kornwachs, K. 2004 « Technik - System - Verantwortung. Eine Einführung », in K. Kornwachs dir. Technik - System - Verantwortung, Münster etc. : LIT Verlag : 23-43.

- dir. 2000 Technikphilosophie, 24 vol., Münster etc. : LIT Verlag.

Krämer, S. \& H. Bredekamp 2009 [2003] « Kultur, Technik, Kulturtechnik : Wider die Diskursivierung der Kultur », in S. Krämer \& H. Bredekamp dir. Bild - Schrift -Zahl, Munich : Fink : 11-22.

Krämer, S. 2004 « Technik als Kulturtechnik. Kleines Plädoyer für eine kulturanthropologische Erweiterung des Technikkonzeptes ", in K. Kornwachs dir. Technik - System - Verantwortung, Münster etc. : LIT Verlag : 157-164.

Kris E. \& O. Kurz 2010 [éd. all. 1934] La Légende de l'artiste. Un essai historique, Paris : Allia.

Krüger, M. 2007 Das Relief der Farbe. Pastose Malerei in der französischen Kunstkritik. 1850-1890, Munich \& Berlin : Deutscher Kunstverlag : 197-227.

- 2012 « Gespachtelter Zufall. Gustave Courbet und die Messermalerei », in P. Cordez \& M. Krüger dir. Werkzeuge und Instrumente, Berlin : Akademie Verlag (« Hamburger Forschungen zur Kunstgeschichte 8 ») : 109-127.

Kubler, G. 1973 [éd. angl. 1962] Formes du temps. Remarques sur l'histoire des choses, Paris : Éditions Champ libre.

Latour, B. \& P. Lemonnier 1994 «Introduction. Genèse sociale des techniques, genèse technique des humains ", in B. Latour \& P. Lemonnier dir. De la préhistoire aux missiles balistiques. L'intelligence sociale des techniques, Paris : La Découverte : 9-24.

Latour, B. \& P. Weibel 2005 Making Things Public : Atmospheres of Democracy, cat. exp. Karlsruhe, Center for Art and Media, Cambridge, Mass. Etc. : The MIT Press.

Lederlin, F. 1997 « L'apparition du terme objet d'art : quelques hypothèses socio-culturelles », French Studies in Southern Africa $26: 33-41$.

Leinenbach, H. 1990 Die Körperlichkeit der Technik. Zur Organprojektionstheorie Ernst Kapps, Essen : Die Blaue Eule.

Lemonnier, P. 1992 Elements for an Anthropology of Technology, Ann Arbor : Museum of Anthropology.

- dir. 1993 Technological Choices. Transformation in Material Cultures Since the Neolithic, Londres : Routledge.

Leroi-Gourhan, A. 1992-1993 [1943-1945] Évolution et techniques, 1, L'Homme et la matière, 2, Milieu et techniques, Paris : Albin Michel.

-1992-1998 [1964-1965] Le Geste et la Parole, 1. Technique et langage, 2. La mémoire et les rythmes, Paris : Albin Michel. 
Martinelli, B. 2005 « Style, technique et esthétique en anthropologie », in B. Martinelli dir. L'interrogation du style. Anthropologie, technique et esthétique, Aix en Provence : Publications de l'Université de Provence : 19-48.

Maurice, K. 1985 Der drechselnde Souverän. Materialien zu einer fürstlichen Maschinenkunst, Zurich : Ineichen.

Mauss, M. 1935 « Les techniques du corps », Journal de Psychologie 32/3-4 : 271-293.

Maye, H. 2010 « Was ist eine Kulturtechnik? », Zeitschrift für Medien- und Kulturforschung 1, Kulturtechnik : 121-135.

Morel, D. 2010 « Archéologie du bâti et anthropologie. L'exemple du technicien dans le Massif central et sur ses marges ( $\mathrm{XI}^{\mathrm{e}}$-XIII ${ }^{\mathrm{e}}$ siècles) », L'Atelier du Centre de recherches historiques. Revue électronique du CRH 6, Faire l'anthropologie historique du Moyen Âge. [En ligne], mis en ligne le 19 juin 2010. URL : journals.openedition.org/acrh/2746. Consulté le 20 décembre 2017.

Muthesius, S. 2001 « Alois Riegl : Volkskunst, Hausfleiss und Hausindustrie », in R. Woodfield dir. Framivng Formalism. Riegls'Work, Londres \& New York : Routledge : 135-150.

Olbrich, H. 1999 Die Casa Zuccari in Florenz : Genese und Erscheinung eines Künstlerhauses der Renaissance, thèse de doctorat, Bamberg.

Osborne, R. \& J. Tanner dir. 2007 Art's Agency and Art History. New Interventions in Art History, Oxford : Wiley-Blackwell.

Pfisterer, U. 2007 « Altamira - oder Die Anfänge von Kunst und Kunstwissenschaft », Vorträge aus dem Warburg-Haus $10: 13-80$.

Pommier, E. 2007 Comment l'art devient l'Art dans l'Italie de la Renaissance, Paris : Gallimard.

Poplin, F. 2012 « L'outil, la matière et la main dans la profondeur de l'esprit », in P. Cordez \& M. Krüger dir. Werkzeuge und Instrumente, Berlin : Akademie-Verlag : 21-32.

Praetorius, M. 1958 [1619] Syntagma Musicum, 2, De Organographia, éd. W. Gurlitt, Kassel etc. : Bärenreiter.

Quiccheberg, S. 2000 [1565] Der Anfang der Museumslehre in Deutschland. Das Traktat «Inscriptiones vel tituli theatri amplissimi » von Samuel Quiccheberg, éd., trad. et comm. H. Roth, Berlin : Akademie-Verlag.

Raff, T. 1994 Die Sprache der Materialien. Anleitung zu einer Ikonologie der Werkstoffe, Münster \& Munich : Waxman.

Raml, M. M. 2009 Der « homo artificialis » als künstlerischer Schöpfer und künstliches Geschöpf. Gentechnologie in Literatur und Leben, Wurzbourg : Königshausen \& Neuman.

Rammert, W. 2007 Technik - Handeln - Wissen. Zu einer pragmatistischen Technik- und Sozialtheorie, Wiesbaden : VS Verlag für Sozialwissenschaften.

Rammert W. \& C. Schubert dir. 2006 Technografie. Zur Mikrosoziologie der Technik, Francfort sur le Main : Campus-Verlag.

Raynaud, C. 2002 « À la hache!» Histoire et symbolique de la hache dans la France médiévale (XIII ${ }^{e}-$ $\mathrm{XV}$ esiècles), Paris : Le Léopard d'Or.

Restle, C. 2006 « Organologie. Die Kunde von den Musikinstrumenten im 17. Jahrhundert », in H. Schramm, L. Schwarte \& J. Lazardzig dir. Instrumente in Kunst und Wissenschaft. Zur Architektonik kultureller Grenzen im 17. Jahrhundert. Berlin : De Gruyter : 277-289. 
Rey, A. dir. 2007 [1992] Dictionnaire historique de la langue française, 3 vol., Paris : Le Robert.

Riegl, A. 1978 [1894] Volkskunst, Hausfleiss und Hausindustrie, Mittenwald : Mäander-Kunstverlags.

- 2014 [1901] L'industrie d'art romaine tardive, Paris : Macula.

Rigaux, D. 2005 Le Christ du dimanche. Histoire d'une image médiévale, Paris : L'Harmattan.

Russo, D. 2008 « Anthropologie et Iconologie. Réflexions sur les apports de Jack Goody à l'analyse de la notion de représentation ", Bulletin du centre d'études médiévales d'Auxerre. Histoire de l'art \& Anthropologie. Études. [En ligne], mis en ligne le 27 février 2008. URL : cem.revues.org/ index4242.html. Consulté le 20 décembre 2017.

Schlanger, N. 1994 «Piaget et Leroi-Gourhan. Deux conceptions biologiques des connaissances et des techniques », in B. Latour \& P. Lemonnier dir. De la préhistoire aux missiles balistiques. L'intelligence sociale des techniques, Paris : La Découverte : 165-186.

Schmitt, J.-C. 1996 « Représentations », in C. Duhamel-Amado \& G. Lobrichon dir. Georges Duby. L'écriture de l'histoire, Bruxelles : De Boeck : 267-278.

Schnapp, A. \& P. Lemonnier 2009 « André Leroi-Gourhan et Pierre Francastel », in T. Dufrêne \& A.-C. Taylor dir. Histoire de l'art et anthropologie. [En ligne], mis en ligne le 28 juillet 2009. URL : journals.openedition.org/actesbranly/80. Consulté le 20 décembre 2017.

Schulz, M. \& B. Wyss dir. 2010 Techniken des Bildes. Munich : Fink.

Schwarz, H.-P. 1990 Das Künstlerhaus. Anmerkungen zur Sozialgeschichte des Genies, Brunswick : Vieweg.

Semper, G. 2007 [manuscrit de 1852] The Ideal Museum. Practical Art in Metals and Hard Materials, éd. P. Noever, Vienne : Schlebrügge.

- 2008 [1860-1863] Der Stil in den technischen und tektonischen Künsten oder Praktische Ästhetik. Ein Handbuch für Techniker, Künstler und Kunstfreunde, 2 vol.. Hildesheim : Olms.

Sennett, R. 2009 [éd. angl. 2008] Ce que sait la main. La culture de l'artisanat. Paris : Albin Michel. Shusterman, R. 2007 [éd. angl. 2008] Conscience du corps. Pour une soma-esthétique. Paris : Éditions de L'Éclat.

Sigaut, F 1987 « Préface. Georges Haudricourt et la technologie », in G. Haudricourt dir. La Technologie science humaine. Recherches d'histoire et d'ethnologie des techniques, Paris: Maison des Sciences de l'Homme : 9-34.

- 1990 « De la technologie à l'évolutionnisme, l'œuvre de Pitt-Rivers », Gradhiva 8 : 20-37.

- 2004 « Les techniques dans la pensée narrative », in J.-L. Jamard dir. Techniques \& Culture 43-44, Mythes. L'origine des manières de faire : 191-214.

-2007 « Les outils et le corps », in T. Pilon \& G. Vigarello dir. Communications 81, Corps et techniques : 9-30.

Simondon, G. 2012 [1958] Du mode d'existence des objets techniques. Paris : Aubier.

Sinkó, K. 2005 « Die Entstehung des Begriffs der Volkskunst in den Kunstgewerbemuseen des Zeitalters des Positivismus : Ornament als Nationalsprache ", Acta historiae artium 46 : 205-259.

Smith, P. H. 2004 The Body of the Artisan. Art and Experience in the Scientific Revolution. Chicago : University of Chicago Press.

Strano, G. et al. dir. 2009 European Collections of Scientific Instruments, 1550-1750. Leyde etc. : Brill. 
Swift, A. 2007 « Soviet Socialism on Display at the Paris and New York World's Fairs, 1937 and 1939 », in H.-J. Czech \& N. Doll dir. Kunst und Propaganda im Streit der Nationen 1930-1945. Dresde : Sandstein : 182-191.

Tarp, L. 2013 « Marble and Marvel : Ole Worm's Globe and the Reception of "Nature's Art" in Seventeenth Century Denmark ", Kritische Berichte 41/3 : 8-23.

Tartuferi, A. 1988 « Le opere d'arte e la decorazione pittorica della chiesa », in F. Gurrieri, L. Berti \& C. Leonardi dir. La Basilica di San Miniato al Monte a Firenze. Florence : Giunti etc : 183-214.

Trischler, H. 2006 « Das Technikmuseum im langen 19. Jahrhundert: Genese, Sammlungskultur und Problemlagen der Wissenskommunikation », in B. Graf \& H. Möbius dir. Zur Geschichte der Museen im 19. Jahrhundert 1789-1918. Berlin : G. \& H. Verlag : 81-92.

Vasari, G. 1981-1989 [éd. it. 1568] Les Vies des meilleurs peintres, sculpteurs et architectes, trad. et éd. comm. sous la dir. d'A. Chastel, 12 vol., Paris : Berger-Levrault.

Vasold, G. 2004 Alois Riegl und die Kunstgeschichte als Kulturgeschichte. Überlegungen zum Frühwerk des Wiener Gelehrten, Fribourg en Brisgau : Rombach.

Wagner, M. 2001 Das Material der Kunst. Eine andere Geschichte der Moderne, Munich : Beck.

Wagner, M., D. Rübel \& S. Hackenschmidt dir. 2010 [2002] Lexikon des künstlerischen Materials. Werkstoffe der modernen Kunst von Abfall bis Zinn, Munich : Beck.

Warnier, J.-P. 1999 Construire la culture matérielle. L'homme qui pensait avec ses doigts, Paris : Presses Universitaires de France.

Welker, M. 2002 « Die Reichsstadt Nürnberg, ein Zentrum des Schmiedeeisen verarbeitenden Handwerks ", in H. Maué et al. dir. Quasi centrum Europae. Europa kauft in Nürnberg. 1400-1800, Nuremberg : Germanisches Nationalmuseum : 119-125.

Werckmeister, O. K. 2002 « Vera Muchina, Arbeiter und Kolchosbäuerin », Kunsthistorische Arbeitsblätter 2002/2 : 41-50.

Williams, R. 1997 Art, Theory, and Culture in Sixteenth-Century Italy. From Techne to Metatechne, Cambridge : Cambridge University Press.

Windmüller, S. 2004 Die Kehrseite der Dinge. Müll, Abfall, Wegwerfen als kulturwissenschaftliches Problem, Münster etc. : LIT Verlag.

Worm, W. dir. 1655 Museum Wormianum, Leyde : Johan Elzevier.

Zimmerli, W. C. 2004 « Technik und Philosophie - 125 Jahre, und wie weiter ? ", in K. Kornwachs dir. 2004 Technik - System - Verantwortung, Münster etc. : LIT Verlag : 665-678.

\section{NOTES}

1. «Outil » dérive d'utensilia et renvoie à l'idée d'utilité, « instrument " procède d'instrumentum : Rey 2007 [1992], art. «outil» et «instrument». Pour l'allemand, Grimm et al. 1854-1960, art. «Instrument » et « Werkzeug ».

2. Sur l'articulation de ces deux notions dans la critique artistique française: Krüger 2007 : 206-208. Voir aussi Krüger 2012.

3. Sur l'histoire des images participant de techniques cognitives: Bredekamp, Bruhn \& Werner 2003 ; Bredekamp, Schneider \& Dünkel 2015 [2008]. Voir aussi Schulz \& Wyss 2010.

4. Quiccheberg 2000 [1565]: 60-69. Avec ce traité, l'auteur proposait ses services au duc de Bavière. 
5. Aujourd'hui au Musée national de la Renaissance à Écouen. Sur Leonhard Danner, son banc de tréfilage et son invention du "bélier à vis » vers 1550, lui aussi présent à la Kunstkammer de Dresde et que Quiccheberg mentionne dans la catégorie des instrumenta violenta, voir Welker 2002. Sur l'ennoblissement de l'artisanat à partir du $\mathrm{XvI}^{\mathrm{e}}$ siècle: Maurice 1985. Sur la Kunstkammer de Dresde, constituée surtout d'instruments scientifiques et d'outils, Korey 2002/2003.

6. Praetorius 1958 [1619] ; voir Restle 2006 ; Gétreau 2006 \& 2007.

7. Voir Heidelberger 2006. Également : Grob \& Hooijmaijers 2006 ; Strano et al. 2009.

8. Voir aussi sur la virtuosité opérationelle Smith 2004.

9. L'objet fut publié dans le catalogue de la collection (Worm 1655), puis donné avec elle, après la mort de Worm, au roi de Danemark. Il se trouve aujourd'hui au château de Rosenborg à Copenhague. Cf. Tarp 2013.

10. Aujourd'hui à la Galleria Estense de Modène, avec d'autres instruments de musique en marbre. Voir Bernardini 2005.

11. Diderot \& D'Alembert 1751-1772. Voir Holländer 2000.

12. Sur les musées de technique et de science, Trischler 2006.

13. Voir Lederlin 1997.

14. Les termes Geräthschaften et Kunstwerke («œuvres d'art») sont notamment utilisés parallèlement par Becker \& Hefner-Alteneck 1852-1863.

15. Semper 2008 [1860-1863]. Voir aussi id. 2007 [1852]. Pour un bilan historiographique: Conforti 1997.

16. Riegl 1978; à ce sujet Muthesius 2001; Vasold 2004. Voir aussi Sinkó 2005. Sur le « Kunstwollen » : Riegl 2014 [1901].

17. Voir Korff 2012 et de manière plus générale id. 2003.

18. Voir Focillon 2013 [1934] ; Ducci 2006.

19. Kubler 1973 [1962]. Cf. aussi quelques années plus tard, Baudrillard 1968, un essai de description sémiologique complète de la culture matérielle contemporaine dans la société de consommation.

20. Boucher de Perthes 1847-1864. Sur les conséquences de la découverte de la préhistoire pour l'histoire de l'art : Pfisterer 2007.

21. Sigaut 1990.

22. Kapp 2007 [1877], cf. Leinenbach 1990.

23. Mauss 1935. Voir aussi Sigaut 2007.

24. Leroi-Gourhan 1992-1993 [1943-1945] ; id. 1992-1998 [1964-1965]. Voir Audouze \& Schlanger 2004. Pour une analyse nuancée de l'évolutionisme de Leroi-Gourhan et sur le concept d'évolution technique, Schlanger 1994 ; Guchet 2008.

25. Cf. la présente revue (depuis 1983). Voir Sigaut 1987 ; Balfet 1991 ; Lemonnier 1992 ; id. 1993 ; Cresswell 1996, et d'un point de vue avant tout historique, la revue Technology and Culture. The International Quarterly of the Society for the History of Technology, 1959.

26. Pour une collaboration entre anthropologues et sociologues de la technique, voir Latour \& Lemonnier 1994, en particulier Akrich 1994. Voir aussi Rammert \& Schubert 2006 ; Rammert 2007.

27. Pour la France voir Warnier 1999 ; Julien \& Warnier 1999 ; Julien \& Rosselin 2005 ; id. 2009.

28. Voir Sennett 2009 [2008]; Crawford 2010 [2009]. Voir aussi la conscience du corps Shusterman 2007 [2008].

29. Latour \& Weibel 2005.

30. Celle-ci a surtout considéré les techniques contemporaines: Hubig, Huning \& Ropohl 2013 [2000] ; Irrgang 2001-2002, et la collection éditoriale Technikphilosophie : Kornwachs 2000-.

31. Voir Kornwachs 2004 et Zimmerli 2004. 
32. Pour une rencontre entre histoire de l'art et anthropologie (pas seulement de l'art) : Dufrêne \& Taylor 2009.

33. Rey 2007 [1992], art. « esthétique » et « technique ».

34. Voir en ce sens, du point de vue de l'histoire de l'art, l'esquisse pionnière de Braunfels 1961, issue d'une conférence à l'Université technique de Rhénanie-Westphalie à Aix-la-Chapelle.

35. Francastel 1988 [1956]. Sur la sociologie des «techniques figuratives» depuis le Quattrocento, Francastel 1989 [1970]. Cf. Schnapp \& Lemonnier 2009.

36. Voir par exemple Bessac 1986 ; Morel 2010. Voir également David 1997.

37. Gell 2009 [1998]. Voir Osborne \& Tanner 2007.

38. Belting 2004 [2001].

39. Voir Krämer 2004 ; Krämer \& Bredekamp 2009 ; Maye 2010.

40. Cf. la collection éditoriale Hengartner 2007-.

41. Cf. l'étude des formes de la socialisation de la «nature» que Descola 2005 a d'abord développée sur un plan cognitif avant tout, avant une proposition d'application au sens d'une « anthropologie de la figuration » : id. 2010.

42. Voir le bilan de recherche et la proposition d'une "définition processuelle du style" de Martinelli 2005, et sur le rapport entre style et outil, D'Onofrio 2005. Autre bilan de recherche anthropologique dans Schnapp \& Lemonnier 2009.

43. Sur l'anthropologie historique à la manière française, voir Brilli, Dittmar \& Dufal 2010.

44. Sur cette discussion, voir par exemple Guille-Escuret 2003, Cresswell 2003.

45. Sur les représentations techniques sémantiques et narratives, Jamard 2004; D'Onofrio \& Joulian 2006 ; Poplin 2012.

46. Windmüller 2004. Pour une autre étude conjointe de techniques et de représentations, Raynaud 2002.

47. Voir Karafyllis 2000 ; id. 2004. Également Flichy 2001.

48. Voir Raml 2009. Sur l'intégration culturelle des « objets techniques » : Simondon 2012 [1958].

49. La construction de la coupole eut lieu entre 1418 et 1436. Le travail lors de jour fériés est attesté treize fois entre 1420 et 1426 pour des raisons diverses : Haines 2002, mots-clés festivas, festivis, festivo. Cf. aussi Braunfels 1965: 218: l'auteur d'une monographie sur l'urbanisme médiéval toscan (id. 1979 [1953]) ne connaît aucune autre permission de travailler le dimanche pour le Quattrocento italien. La datation de l'image par sa fonction rejoint son attribution par le style à l'œuvre précoce de Mariotto di Cristofano (1393-1457) : cf. Tartuferi 1988 : 208.

50. Voir Rigaux 2005 : 99, 154 \& 302-303 sur cette image (avec des interprétations erronées de la technique et de l'état de conservation).

51. L'œuvre est aujourd'hui exposée à Moscou. Je remercie Monika Wagner et nos étudiantes de l'Université de Hambourg pour des réflexions dans le cadre d'un séminaire. Voir Werckmeister 2002 ; Jungen 2005 ; Swift 2007.

52. Sur la sémantique des matériaux dans l'art, voir Raff 1994; pour l'art moderne et contemporain, Wagner 2001 ; Wagner, Rübel \& Hackenschmidt 2010 [2002].

53. Pour un travail pionnier, voir Kris \& Kurz 2010 [1934]. De manière générale sur les récits liés aux techniques, mais sans considération de l'historiographie de l'art, Sigaut 2004.

54. Très usés, les reliefs ont été reconstitués en 2002-2003 et ne peuvent être pleinement tenus pour des témoins historiques. Il n'est pas certain que l'image peinte, dont on fait l'hypothèse à partir d'autres œuvres de Zuccari, ait jamais été réalisée. Voir Acidini 2009.

55. Voir Williams 1997 : 135-150 sur la théorie de Zuccari.

56. Voir Schwarz 1990: 177-182, 194-200; Olbrich 1999: 135-138. Sur le topos de l'atelier d'artiste : Coleu \& Pardo ; Diers \& Wagner 2010.

57. Voir Williams 1997 : 29-72 ; Vasari 1981-1989 [1568].

58. Voir par exemple Pommier 2007. Sur de telles situations d'interactions particulièrement rapides entre le technique et le social, voir Cresswell 1994. 
59. Cf. l'observation que les rapports entre techniques et sociétés seraient plus métaphoriques dans des contextes traditionnels et plus directs dans des contextes industriels, et que l'anthropomorphisme, d'abord projeté ou figuré sur les objets, entrerait ensuite «dans les mécanismes eux-mêmes » : Latour \& Lemonnier $1994: 18$.

\section{ABSTRACTS}

If the terms "tool" and "instrument" have in common the designation of objects that humans fabricate to perform one or more tasks, they nevertheless divide these tasks into two distinct spheres of activity. Tools act directly on the raw material, for purposes of work, and instruments, more finely elaborated, pertain to privileged domains of music, science or art. These two words, taken together, invite examination of the manufacture and the handling of objects, with the aim of better understanding the relations between what one designates as "art" and what one conceives as "technique" in Western societies.

This then raises the question of the relationship between art history and the anthropology of techniques, in particular the contribution of the former to the latter. The present essay's approach is historiographic - with particular attention to research within the German-speaking world - and at the same time prospective. It considers a vast spectrum of tools and historical instruments that - despite being elaborate objects, associated with particular expertise, and collected since the sixteenth century - are not works of art in the usual sense of the term. In their materials and their forms, tools and instruments participate in processes that are at once artistic and technical. They are likewise involved at the level of their representations, and play a major role in the socialization of these activities, that is to say, ultimately in the contribution of arts and techniques to the symbolic production of societies. This is demonstrated in three image studies, ranging from the fifteenth to the twentieth century. Situating the notion of art anthropologically by observing its links with the history of the techniques reinforces the participation of the history of the art as well as the anthropology of the techniques in the collective enterprise of the social sciences.

A first version of this text was published in German in a collection on « Tools and Instruments » (Cordez 2012). Translation by Naïma Ghermani and the author.

Si les termes « outil » et « instrument » ont en commun la désignation des objets que les humains fabriquent en vue d'exécuter une ou plusieurs tâches, ils répartissent néanmoins ces dernières en deux champs d'activités distincts. Les outils agissent directement sur la matière brute, pour travailler, et les instruments, plus finement élaborés, ont la préférence des domaines privilégiés de la musique, de la science ou de l'art. Ces deux mots, mis en pendant, invitent donc à l'étude de la fabrication et du maniement des objets, en s'efforçant de mieux appréhender les relations entre ce que l'on désigne comme "art » ou ce que l'on conçoit comme «technique », dans les sociétés occidentales.

Se pose alors la question des rapports entre l'histoire de l'art et l'anthropologie des techniques, en particulier de l'apport de la première à la seconde. L'approche de cette contribution est à la fois historiographique - avec une attention particulière pour les recherches germanophones - et prospective. Il s'agit d'abord de considérer un vaste spectre d'outils et d'instruments historiques qui ne sont pas des œuvres d'art au sens usuel du terme, bien qu'étant des objets élaborés, liés à des savoir-faire particuliers, et collectionnés depuis le $\mathrm{XVI}^{\mathrm{e}}$ siècle. Par leurs matières et par leurs 
formes, outils et instruments participent de processus, tout à la fois artistiques et techniques. Ils sont de même impliqués dans leurs représentations, et jouent de ce fait un rôle majeur dans la socialisation de ces activités, c'est-à-dire finalement dans la contribution des arts et des techniques à la production symbolique des sociétés. C'est ce qui apparaît dans trois études d'images, allant du $\mathrm{xv}^{\mathrm{e}}$ au $\mathrm{xx}^{\mathrm{e}}$ siècle. Situer anthropologiquement la notion d'art en observant ses liens avec l'histoire des techniques renforce la participation tant de l'histoire de l'art que de l'anthropologie des techniques à l'entreprise collective des sciences sociales.

Une première version de ce texte est parue en allemand dans un volume collectif sur les «Outils et instruments » (Cordez 2012). Traduction de Naïma Ghermani et de l'auteur.

INDEX

Keywords: art, technique, object, tool, instrument, representation.

Mots-clés: art, technique, objet, outil, instrument, representation.

\section{AUTHOR}

\section{PHILIPPE CORDEZ}

Directeur adjoint, Centre allemand d'histoire de l'art, Paris. 